\title{
Electric field penetration effects in time-of-flight measurements
}

\author{
U.K. Abeywarna ${ }^{a} *$ T.R. Ariyaratne ${ }^{b}$ \\ ${ }^{a}$ Department of Physics, University of Kelaniya, Kelaniya, Sri Lanka \\ ${ }^{b}$ Department of Physics, University of Colombo, Colombo, Sri Lanka
}

\begin{abstract}
The field imperfections in the mass spectrometers were studied by means of two Plasma desorption time-of-flight mass spectrometers. The experiment was performed by investigating the influence of the field leakage due to the post acceleration on the measured values of the initial axial energy of secondary ions, in the presence of the grounded grids, which are usually employed to separate the fields inside the mass spectrometers. It has been found that the changes of $0.4 \%$ in the effective lengths of the acceleration gap and the field free region respectively are possible due to the field penetration into those regions from the post acceleration gap of the mass spectrometer. The analyses also show clearly the significance of the influence of the field penetration of the post acceleration on the measured values of the initial axial energy. The initial axial energies of $\mathrm{H}^{+}, \mathrm{H}_{2}{ }^{+},{ }^{6} \mathrm{Li}^{+},{ }^{7} \mathrm{Li}^{+}, \mathrm{Na}^{+}$and $\mathrm{Cs}^{+}$measured under the minimum field penetration condition were found to be $4.7 \mathrm{eV}, 4.7 \mathrm{eV}, 0.6 \mathrm{eV}, 0.7$ $\mathrm{eV}, 0.7 \mathrm{eV}$ and $1.3 \mathrm{eV}$ respectively, and they had been reduced to $4.4 \mathrm{eV}, 4.4 \mathrm{eV}, 0.3 \mathrm{eV}, 0.4$ $\mathrm{eV}, 0.4 \mathrm{eV}$ and $1.0 \mathrm{eV}$ once the values were corrected separately for field penetration. These results indicate that the field penetration through a grid is a secondary effect and it is hardly unavoidable in the mass spectrometers.
\end{abstract}

\section{INTRODUCTION}

Ion optical properties associated with electric fields play an important role in mass spectrometers as most of them consist of at least one of the ion optic systems such as gaps with constant accelerating and post accelerating fields, Einzel lenses, deflection plates, and ion reflectors ${ }^{1,2}$. These ion optic systems are expected to provide required effects while confining their electric fields to the respective geometrical spaces occupied by these devices inside the mass spectrometer. However, in reality the electric fields cannot be confined completely to specific regions, and therefore the demarcations of such electric

\footnotetext{
*Corresponding Author, E-mail: kanthi@kln.ac.lk
} 
fields are not properly defined in mass spectrometers. This results in the field penetration and the field inhomogeneity inside mass spectrometers ${ }^{2,3}$.

In time-of-flight mass spectrometers, the time-of-flight (TOF) of an ion is a direct experimental observation, which can be accumulated to generate a TOF spectrum, and later analysed to extract parameters such as mass, velocity and energy of the ion. Fig. 1 shows the various regions through which an ion travels from its generating point till it reaches the stop detector.

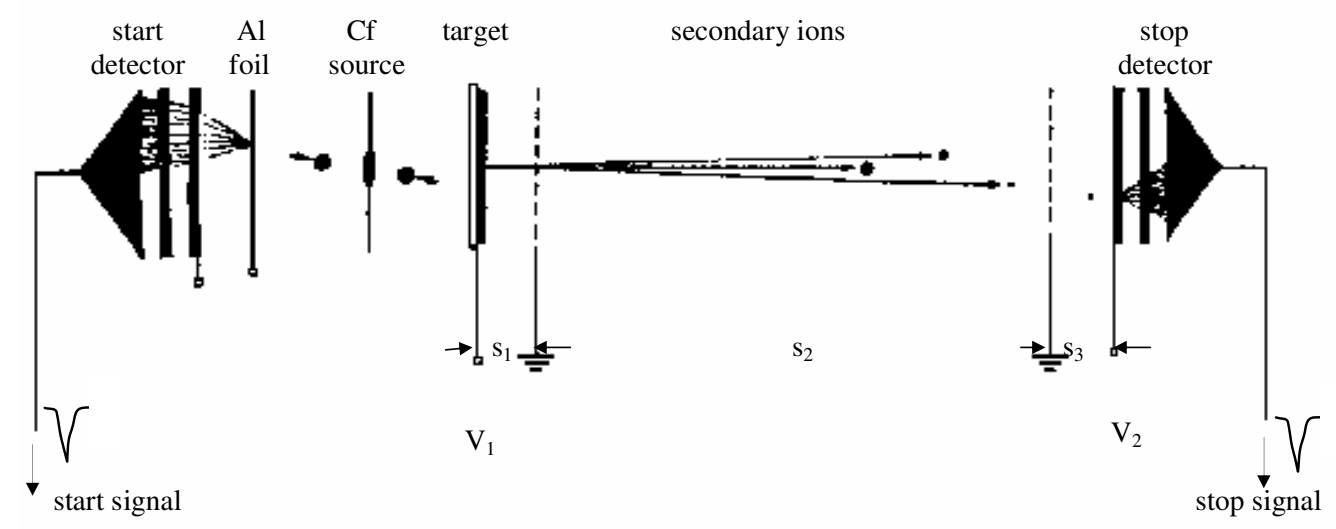

Figure 1. A schematic diagram of a plasma desorption TOF mass spectrometer. $s_{1}, s_{2}$ and $s_{3}$ are the lengths of the acceleration region, the field-free region and the post acceleration region respectively; $\mathrm{V}_{1}$ and $\mathrm{V}_{2}$ are the acceleration and post acceleration voltages respectively.

The TOF of an ion depends on the ion path, which is determined by the electric field lines present in the mass spectrometer. Hence, the field penetration through the grids, which are used to separate various electric field regions inside a mass spectrometer, is a prominent factor that affects the TOF of an ion. It also results in a divergence of the secondary ion away from the expected path. The divergence of a secondary ion depends on the grid size and the wire diameter of the grid ${ }^{4}$. Furthermore, it has been shown that a smaller grid size and a smaller wire diameter reduce the beam divergence. However, the TOF equation that is used to extract various parameters related to the ion is generally derived assuming that the applied electric fields are uniform, and well confined to the respective regions ${ }^{5}$. Hence the field penetration effects due to the grids are not included in the derivation of the TOF equation which is used to analyse most of the experimental observations. In most of the mass spectrometric studies the field penetration is usually considered as a second order effect, which introduces errors into such measurements. However, when making precise measurements on parameters whose magnitudes themselves are comparable with such errors, then those effects need to be taken in to account. In general when a transparent grid is used to separate two electrostatic field regions, it modifies the applied electric field on either side of the grid $^{6,7}$ due to the field penetration, and produces 'localized microlensing effects $^{\prime 8}$ at the proximity of the grid openings. Such field penetrations give rise to an average effective potential on the grid which is different from the applied potential to the grid. This change in the effective potential in turn has the effect of changing the shortrange as well as long-range fields and potentials on both sides of the grid $^{7}$. 
In this paper, we report a study of such effects by investigating the influence of the field leakage from the post acceleration grid employed in a Plasma desorption TOF mass spectrometer (PDMS) on the measured values of the initial axial energy ${ }^{5}$ of secondary ions.

\subsection{Initial axial energy}

The secondary ions in a PDMS are formed by bombarding a solid sample surface with fast heavy ions from a ${ }^{252} \mathrm{Cf}$ radioactive source. The term, initial kinetic energy, is used to represent the energy acquired by such secondary ions during the desorption process. The word 'initial' is used here to differentiate this kinetic energy from the kinetic energy which is subsequently acquired by the desorbed ion, when it is accelerated to high velocities for detection purposes. The measurement of this initial energy is immensely helpful in the understanding of the mechanism behind the desorption process. There are two basic experimental approaches to determine the initial kinetic energy; one is through the measurements of initial 'axial' energy and the other through measurements of initial 'radial' energy. The energy measurements performed on the initial velocity component in the direction perpendicular to the plane of the target surface and along the axis of the mass spectrometer are called the initial axial energy measurements. In this nomenclature the term radial energy is used to represent the total radial energy itself.

The initial axial energy is only a process dependent parameter and hence it should be independent of the evaluation method. Furthermore the direct measurement of the initial axial energy of a particular ion with significant accuracy is not directly achievable. However, an indirect method which uses TOF equation of the TOF mass spectrometer and the time-of-flight measurement has been developed ${ }^{5,9}$ to measure this parameter with an accuracy of $0.1 \mathrm{eV}$. This method which is adopted in the study is described elsewhere ${ }^{5}$, and it provides a way of determining the initial axial energy of secondary ions, after evaluating the mass spectrometer constants. The TOF equation mentioned above considers an ideal situation and does not make any allowance for field leakage from region to region.

The expression for the total TOF for such an ion, i.e. the TOF equation, is given in Eq. 1,

$$
\mathrm{TOF}=\sqrt{\frac{2 \mathrm{~m}}{\mathrm{q}}}\left(\frac{\mathrm{s}_{1}}{\mathrm{~V}_{1}}\left(\sqrt{\mathrm{V}_{1}+\frac{\mathrm{E}_{\mathrm{a}}}{\mathrm{q}}}-\sqrt{\frac{\mathrm{E}_{\mathrm{a}}}{\mathrm{q}}}\right)+\frac{\mathrm{s}_{2}}{2 \sqrt{\mathrm{V}_{1}+\frac{\mathrm{E}_{\mathrm{a}}}{\mathrm{q}}}}+\frac{\mathrm{s}_{3}}{\mathrm{~V}_{2}}\left(\sqrt{\mathrm{V}_{2}+\mathrm{V}_{1}+\frac{\mathrm{E}_{\mathrm{a}}}{\mathrm{q}}}-\sqrt{\mathrm{V}_{1}+\frac{\mathrm{E}_{\mathrm{a}}}{\mathrm{q}}}\right)\right)+\mathrm{T}_{0}, \ldots(1)
$$

Where $\mathrm{m}$ and $\mathrm{q}$ are the mass and the charge of the desorbed secondary ion and $\mathrm{T}_{0}$ is the time off-set. $\mathrm{T}_{0}$ includes the propagation delays due to the electronics, delays occurring in the start and stop detectors, and also the time difference between the actual desorption event and the starting time of the TOF measurement. $E_{a}$ is the initial kinetic energy corresponding to the initial axial velocity component and it is the initial axial energy. The distances, $\mathrm{s}_{1}, \mathrm{~s}_{2}, \mathrm{~s}_{3}$ and $\mathrm{T}_{0}$ are generally known as mass spectrometer constants.

If a field penetration occurs across the boundaries of the various flight regions, the effective values of s1, s2 and s3 may be different from the physical dimensions. These 
quantities are called the effective distances and cannot be measured directly. Under this situation, the initial axial energy $E_{a}$ value determined after substituting the experimentally observed TOF values in Eq. 1 will have a small perturbation due to the field leakage. The observed $E_{a}$ value depends on the magnitude of the field leakage, and therefore can be used as a parameter to study the field leakage inside a TOF mass spectrometer, and thereby to test the designs of various ion optic components for field leakages.

\section{EXPERIMENTAL}

\subsection{Instrumentation}

The initial axial energy measurements were performed using a linear ${ }^{252} \mathrm{Cf}$ plasma desorption TOF mass spectrometer ${ }^{5}$ at the Department of Physics of the University of Colombo, Sri Lanka, and a modified BIOION 20 TOF mass spectrometer ${ }^{5}$ at the Division of Ion Physics, the Angström Laborotary, Uppsala University, Sweden. A schematic diagram of linear ${ }^{252} \mathrm{Cf}$ plasma desorption TOF mass spectrometer is shown in Fig. 1. The operational features of two mass spectrometers are similar but the mass spectrometer constants ${ }^{5}$ associated with them are different. Both mass spectrometers were operated only with acceleration and post acceleration voltages on while keeping all the other ion optic systems switched off.

The TOF mass spectrometer basically consists of a ${ }^{252} \mathrm{Cf}$ radioactive source ejecting high energy fission fragments to produce molecular ions from a target, an acceleration gap, a field free drift region, a post acceleration gap for ions and micro channel plate ion detectors to provide time markers for the start and stop of a TOF event. As the fission fragments are produced in pairs and move in opposite directions, one of the fragments in the pair is used to generate a pulse and set the start time marker (see Fig. 1).

A positive voltage (V1) was applied to the target in order to accelerate secondary ions of positive polarity towards the field free region. This voltage was provided by an extremely stable and calibrated DC standard voltage supply (model 2552 - Yokogawa), and the acceleration voltage was kept at low values $(\leq 300 \mathrm{~V})$ in order to observe initial axial energy distributions of ions more clearly. The stop detector in the mass spectrometer was operated with a negative post acceleration voltage to ensure efficient ion detection. Standard NIM electronics and a ATARI Mega ${ }^{\text {ST4 }}$ PC were used for the data acquision.

The stop detector section of the mass spectrometer in Colombo was reconstructed ${ }^{5}$ for this purpose in such a way that it minimises the possible field leakage into the field free region except the unavoidable field penetration through the grounded grid of the stop detector (Fig. 2). However, that too was reduced to some extent by mounting a second grounded grid in front of the first grid. 


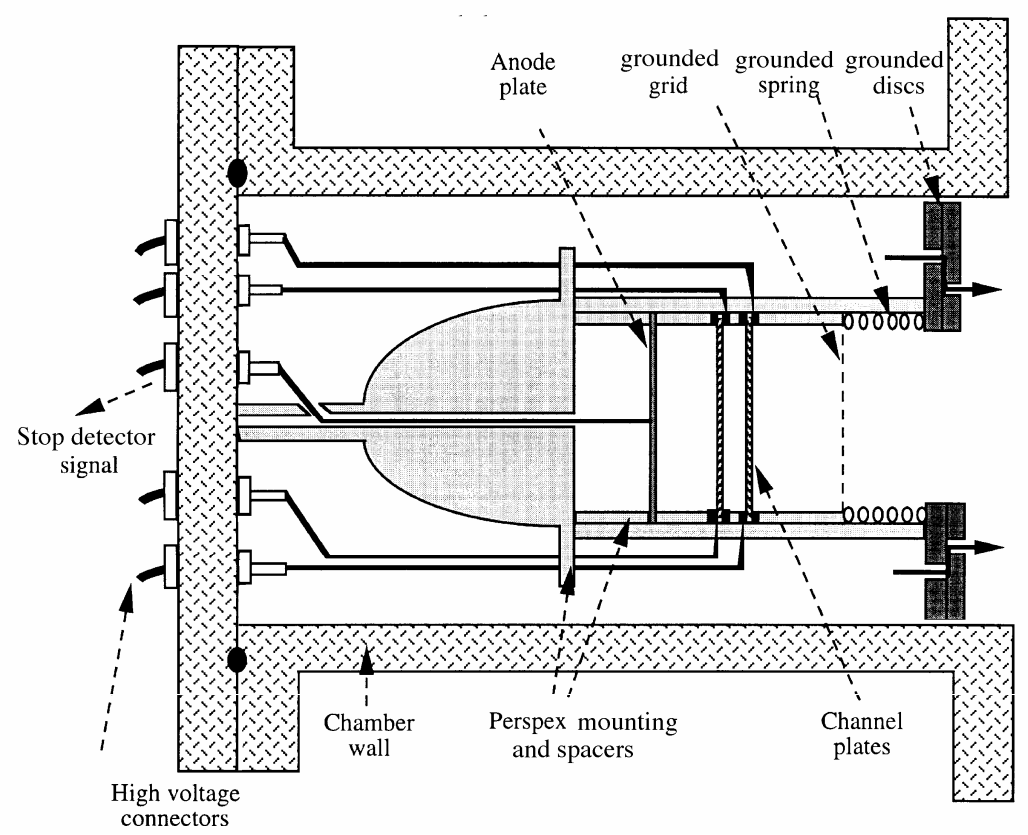

Figure 2. The stop detector in Colombo after modification

To minimise the field leakage into the field-free region from the high voltage side of the stop detector, two grounded metal discs were fixed just above the stop detector, screening the high voltage section. The external diameter of the grounded metal discs was chosen so as to cover the high voltage side of the stop detector. It had several holes of $5 \mathrm{~mm}$ diameter in order to facilitate the evacuation process. The holes were made in such a way that no field lines could get through to the field free region via them. In this configuration, the secondary ions that reach the stop detector can hardly be influenced by the fields other than those penetrating through the grounded grids. The modification of the stop detector section in this way helped to minimise the field leakage into the field free region considerably.

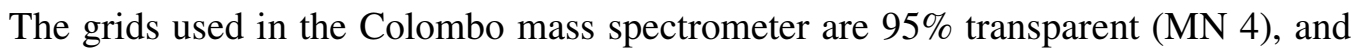
the wire diameter and the separation between two adjacent wires are $0.0322 \mathrm{~mm}$ and $1.21 \mathrm{~mm}$ respectively.

Initial energy measurements were also carried out with the Uppsala mass spectrometer mentioned above after carrying out similar modifications to its stop detector. The grids used in this mass spectrometer are $90 \%$ transparent (MN 17), and the wire diameter and the wire separation are $0.0185 \mathrm{~mm}$ and $0.34 \mathrm{~mm}$ respectively.

In order to study the effects of the field imperfections inside the mass spectrometers, the post acceleration voltage was varied between $-2550 \mathrm{~V}$ and $-6500 \mathrm{~V}$ (between - $2336 \mathrm{~V}$ and $-4957 \mathrm{~V}$ in the Uppsala mass spectrometer) by using a variable potential divider while monitoring the constant potential difference across the channel plates. 
Sets of spectra for secondary ions of $\mathrm{H}^{+}, \mathrm{H}_{2}{ }^{+},{ }^{6} \mathrm{Li}^{+},{ }^{7} \mathrm{Li}^{+}, \mathrm{Na}^{+}$and $\mathrm{Cs}^{+}$were accumulated for the positive acceleration voltage range from $200 \mathrm{~V}-300 \mathrm{~V}$ in $20 \mathrm{~V}$ steps for each post acceleration voltage for both instruments. The centroid value of each secondary ion peak was considered as the TOF value of each secondary ion and was extracted from the accumulated spectra. TOF vs. the acceleration voltage graphs were plotted for each post acceleration voltage and the mass spectrometer constants were then evaluated using an analytical method ${ }^{5}$. Subsequently the TOF equation was fitted using the known values of mass spectrometer constants in order to obtain the initial axial energy.

\subsection{Sample preparation}

As the targets are bombarded from backside by the fission fragments of ${ }^{252} \mathrm{Cf}$ source, the samples were prepared on thin aluminised Mylar backings of thickness $300 \mu \mathrm{g} / \mathrm{cm}^{2}$. In order to obtain a fairly good homogeneous target surface, the spin coating method ${ }^{10}$ has been used for the preparation of all targets. Targets were prepared by using a solution, which contains $\mathrm{LiCl}, \mathrm{NaCl}$ and $\mathrm{CsBr}$. The amount of the sample solution, the speed of the spinning and the height from the dropper are always kept constant in order to maintain the thickness of the target layer approximately the same in each case. All the compounds used in this study were purchased from Sigma Chemical Company, St. Louis, USA.

\section{RESULT AND DISCUSSION}

The two sets of measurements employing Colombo and Uppsala mass spectrometers were performed independently after minimising the possible field leakage from the stop detector sections as described above. Field penetrations to the flight region from the start detector is considered negligible, as the latter is located away and well shielded due to the presence of grounded metal parts inside the mass spectrometer. After analysing the mass spectrometer constants, the initial axial energy values were extracted for different post acceleration voltages, which act as the main 'source' of field leakage. It should be noted that the field penetration from the acceleration grid to the field free region is less at least by a factor of five as the acceleration field was kept at low values compared to the post acceleration field.

Fig. 3 shows the initial axial energy variation with the post acceleration voltage for five different secondary ions desorbed from the same target. (a) - graphs represent the results obtained with the Colombo mass spectrometer, and (b) - graphs show the corresponding values for the Uppsala mass spectrometer. 

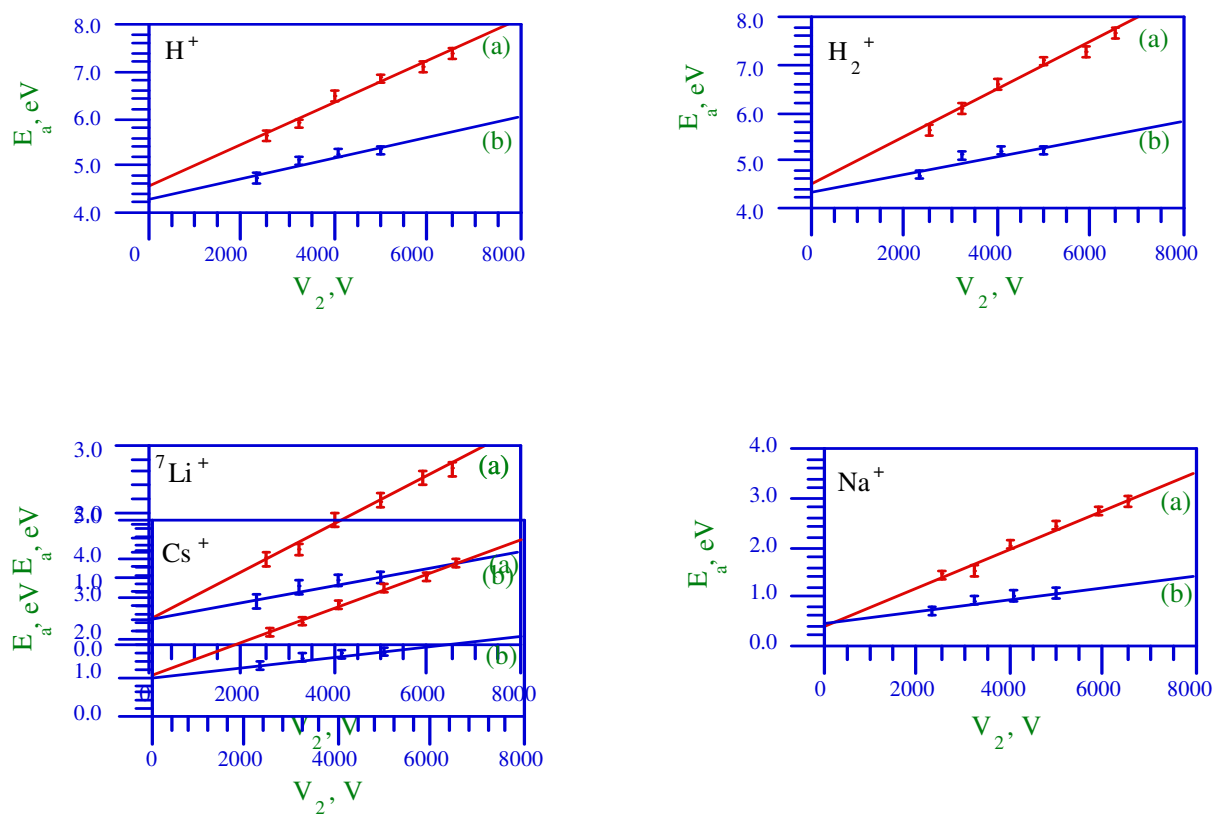

Figure 3. The variation of the initial axial energy values with the post acceleration voltage for $\mathrm{H}^{+}, \mathrm{H}_{2}^{+},{ }^{7} \mathrm{Li}^{+}$, $\mathrm{Na}^{+}$and $\mathrm{Cs}^{+}$ions. Graphs are drawn for the experimental results obtained (a) in Colombo and (b) in Uppsala

All the curves exhibit a variation of the initial axial energy values with the post acceleration voltage and it is apparent that the observed variation shows a linear behaviour at least for the investigated voltages (see Fig. 3). However, in the case of Uppsala results a slight tendency for saturation at high post acceleration is apparent, but the accuracy of the results is not good enough to arrive at any conclusion.

The gradient of each line can be considered as a measure of the strength of the field leakage due to the stop detector section. The gradient of the plots obtained with the Colombo mass spectrometer is found to be greater than the corresponding values obtained with the Uppsala system implying a higher field leakage from the Colombo set up. The main reason for this difference may be due to the transparency of the grids being used in the stop detectors. Fig. 4 shows the enlarged pictures of the two grids used in the two occasions, after magnifying by a constant factor. The screening effect of the 'Uppsala grid' is obviously higher compared to the 'Colombo grid'.
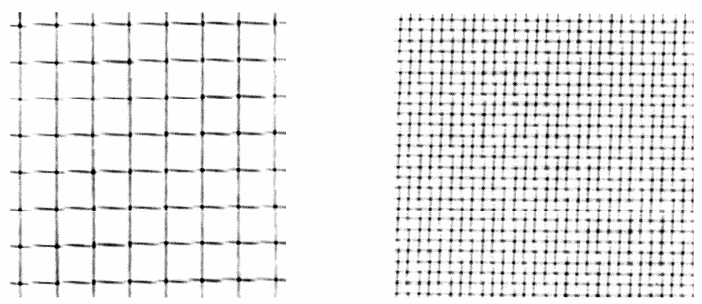

Figure 4. The enlarged pictures of the grids used in the mass spectrometers in Colombo (left) and Uppsala (right). The real sizes are magnified by a constant factor 
The quantitative measure of the field leakage $\left(\mathrm{V}_{\mathrm{f}}\right)$ associated in each measurement (in terms of volts) can be calculated by using the following expression:

$$
\mathrm{V}_{\mathrm{f}}=\frac{\left(\mathrm{E}_{\mathrm{a}}\right)_{\mathrm{v}}-\left(\mathrm{E}_{\mathrm{a}}\right)_{0}}{\mathrm{q}}
$$

where $\left(E_{a}\right)_{v}$ is the initial axial energy of the ion when there is a post acceleration voltage of $\mathrm{V}_{2},\left(\mathrm{E}_{\mathrm{a}}\right)_{0}$ is the corresponding value at zero post acceleration voltage, and $\mathrm{q}$ is the charge associated with the ion. Field leakage values calculated using the above expression for the Colombo and the Uppsala detector sections are given in Table 1.

Table 1. Comparison of the field leakage in both mass spectrometers (SD and MS refer to the stop detector and the mass spectrometer respectively)

\begin{tabular}{|c|c|c|c|}
\hline $\begin{array}{c}\text { Post acceleration, } \mathrm{V} \\
(\mathrm{SD} \text { in Colombo) }\end{array}$ & $\begin{array}{c}\text { Field leakage }\left(\mathrm{V}_{\mathrm{f}}\right), \\
\mathrm{V} \\
(\text { MS in Colombo) }\end{array}$ & $\begin{array}{c}\text { Post acceleration, } \mathrm{V} \\
(\mathrm{SD} \text { in Uppsala })\end{array}$ & $\begin{array}{c}\text { Field leakage }\left(\mathrm{V}_{\mathrm{f}}\right), \\
\mathrm{V} \\
(\text { MS in Uppsala) }\end{array}$ \\
\hline 2550 & 1.0 & 2336 & 0.3 \\
\hline 3200 & 1.3 & 3234 & 0.5 \\
\hline 4000 & 1.7 & 4106 & 0.7 \\
\hline 4950 & 2.1 & 4957 & 0.8 \\
\hline 5900 & 2.5 & - & - \\
\hline 6500 & 2.7 & - & - \\
\hline
\end{tabular}

The results indicate that the values of the field leakage are extremely small compared to the post acceleration voltages. However, they are comparable with the voltages corresponding to the initial axial energy values, which are shown in Table 3.

Pairs of lines drawn for all the different secondary ions seem to converge on or near the axis yielding $\left(\mathrm{E}_{\mathrm{a}}\right)_{0}$ values (see Fig. 3). The errors in intercepts of the two lines of all the plots except the one for hydrogen are within the acceptable error limits of the initial axial energies of $0.1 \mathrm{eV}$. These values can be considered as the initial axial energies of the secondary ions corrected for the field leakage due to the stop detector.

Table 2 shows the geometrical lengths of the acceleration gap $\left(s_{1}\right)$ and the field free region $\left(s_{2}\right)$ of the Uppsala mass spectrometer together with the effective values of the same derived from the experimental analysis before and after the reduction of the field-leakage being done. There is a significant difference $(5 \%)$ between the geometrical length of $s_{1}$ and its effective value. Major factors that contribute to produce this difference are field penetration, and the field distortion in the acceleration region, due to inhomogeneities in the wire grid surface and the sample thickness. However, in the case of the field free region, the effective length differs from its geometrical length only by $1.3 \%$, and it indicates that the influence of the above factors is significant only when the gap is small. The results also indicate that even after the installation of two grounded grids and a shield in front of the stop detector, the change in the effective lengths of the acceleration gap $\mathrm{s}_{1}$, and the field free region $s_{2}$ achieved are only about $0.4 \%$. These observations also show 
that the field penetration through grids is only a secondary effect and it is hardly unavoidable through grids.

Table 2. Comparison of the length of the field free region before and after the modification of the stop detector section in the mass spectrometer in Uppsala

\begin{tabular}{|c|c|c|}
\hline Geometrical length & $\begin{array}{c}\text { Effective Length before } \\
\text { modifying the stop } \\
\text { detector }\end{array}$ & $\begin{array}{c}\text { Effective Length after } \\
\text { modifying the stop } \\
\text { detector }\end{array}$ \\
\hline $\mathrm{s}_{1}: 4.86 \mathrm{~mm}$ & $5.10 \mathrm{~mm}$ & $5.12 \mathrm{~mm}$ \\
\hline $\mathrm{s}_{2}: 236.8 \mathrm{~mm}$ & $239.85 \mathrm{~mm}$ & $238.90 \mathrm{~mm}$ \\
\hline
\end{tabular}

Table 3 shows the initial axial energy values of six different secondary ions extracted from the analysis before and after the modification of the Uppsala mass spectrometer. The results were obtained using post acceleration voltages of $2336 \mathrm{~V}$ before and after the modification of the stop detector section.

According to Table 3, the initial axial energies of the secondary ions considered are comparatively low after the modification of the stop detector section except for hydrogen ions. The difference is about $35-40 \%$. The main reason for this may be the minimisation of the field leakage from the stop detector section of the mass spectrometer on the latter occasion. Even though the difference between the geometrical length and the effective length of $s_{2}$ corresponding to the field free region is about $1 \mathrm{~mm}$ for both cases, the initial axial energy values have changed by a significant amount. When compared to the changes observed in the mass spectrometer constants, the influence of the post acceleration voltage on the initial axial energy is very significant and is clearly seen also from the curves in Fig. 3. This indicates that the influence of the field penetration affects significantly the initial axial energies deduced from the data but not the secondary ion path lengths.

Table 3. The initial axial energies $\left(\mathrm{E}_{\mathrm{a}}\right)$ of secondary ions desorbed from targets consisting of a mixture of $\mathrm{LiCl}, \mathrm{NaCl}$ and $\mathrm{CsBr}$. The results were obtained with the Uppsala mass spectrometer operated at acceleration voltages of $200 \mathrm{~V}$ to $300 \mathrm{~V}$ and post acceleration voltage of $2336 \mathrm{~V}$ before and after the modification.

\begin{tabular}{|c|c|c|c|}
\hline Secondary ion & $\begin{array}{c}\mathrm{E}_{\mathrm{a}}, \mathrm{eV} \\
\text { (before modification) } \\
\mathrm{V}_{\text {post }}=2336 \mathrm{~V}\end{array}$ & \multicolumn{2}{|c|}{$\begin{array}{c}\mathrm{E}_{\mathrm{a}}, \mathrm{eV} \\
\text { (after modification) }\end{array}$} \\
\cline { 3 - 4 } & & $\mathrm{V}_{\text {post }}=2336 \mathrm{~V}$ & $\mathrm{~V}_{\text {post }}=0$ (corrected) \\
\hline $\mathrm{H}^{+}$ & 4.8 & 4.7 & 4.4 \\
\hline $\mathrm{H}_{2}^{+}$ & 4.7 & 4.7 & 4.4 \\
\hline $6 \mathrm{Li}^{+}$ & 1.0 & 0.6 & 0.3 \\
\hline $7 \mathrm{Li}^{+}$ & 1.2 & 0.7 & 0.3 \\
\hline $\mathrm{Na}^{+}$ & 1.2 & 0.7 & 0.4 \\
\hline $\mathrm{Cs}^{+}$ & 2.0 & 1.3 & 1.0 \\
\hline
\end{tabular}


Considering the field penetration for known acceleration and post acceleration voltages, the initial axial energy values can be corrected for field leakage, and the values thus obtained for secondary ions are also shown in Table 3. These values are much closer to the values obtained in Uppsala with a minimum post acceleration voltage (see Table 3 ). The main difference between the mass spectrometers used for the measurements in Colombo and Uppsala was the mounting of an additional shield and a grid in the latter in order to minimise the field penetration due to the stop detector section. These results show that all stray fields created in the mass spectrometer affect the information extracted from the systems, and should be eliminated in order to obtain a more reliable value for the initial axial energy.

\section{CONCLUSION}

Results indicate that the significant influence of the stray fields created in the mass spectrometer on the measurement on the initial axial energy even after minimizing the possible field leakage in the mass spectrometer. This leads to a general conclusion that the field penetration through a grid is a secondary effect and it is hardly unavoidable in the mass spectrometers.

\section{ACKNOWLEDGEMENT}

Authors greatly acknowledge the International Science Program, Uppsala University for the fellowship provided to UK Abeywarna; and the Department of Radiation Science, Uppsala University and the Department of Physics, University of Colombo for the facilities provided to conduct the research work. It is a grate pleasure to acknowledge the significant contribution of Prof, P.Håkansson to conduct the research work in Uppsala University and his valuable discussion and suggestions during the work. Roman Zubarev and WMKP Wijayaratna are greatly acknowledged for suggestions made during the work. We thank Johan Kjellberg, the Department of Radiation Science, Uppsala University and Sarath Kumara, workshop engineer, in the technical workshop, University of Colombo for their invaluable technical assistantance given throughout the research work. 


\section{REFERENCES}

1. R.D. Macfarlane, J.C. Hill and D.L. Jacob, A new californium 252 plasma desorption TOF mass spectrometerfor high mass studies, Analytical Instrumentation, 16(1), (1987) 51

2. G. Brinkmalm, P. Håkansson, J. Kjellberg, P.Demirev, B.U.R. Sundqvist, and W. Ens, A plasma desorption TOF mass spectrometer with a single stage ion mirror: Improved resolution and calibration procedure, Int. J. Mass Spectrom. Ion Processes, 114, (1992) 183

3. R.A. Zubarev, P.Håkansson, and B.U.R. Sundqvist, Accurate monoisotopic mass measurements of peptides: posibilities and limitations of high resolution TOF particle desorption mass spectrometry, Rapid Commun. Mass Spectrom., 10, (1996) 1386

4. H. Danigel and R.D. Macfarlane, Beam profile analysis for a using ${ }^{252}$ Cf plasmadesorption TOF mass spectrometer, Int. J. Mass Spectrom. Ion Phys., 39, (1981) 157

5. U.K.Abeywarna, Initial energy distributions of secondary ions in electronics sputtering, Ph.D. thesis, University of Colombo, 1999

6. F. H. Read, N. J. Bowring, P. D. Bullivant, and R.R.A.Ward, Penetration of lectrostatic fields and potentials through meshes, grids, or gauzes, Rev. Sci Instr. 69(5), (1998)2000

7. F. H. Read, N. J. Bowring, P. D. Bullivant, R. R. A. Ward, Short-and long-range penetration of fields and potentials through meshes,grids or gauzes, Nucl.Instr.\& Meth. A 427(1999) 363.

8. D. L. Williams, F. H. Read, N. J. Bowring, Defocussing of charged particle beams transmitted through meshes. Nucl. Instr. \& Meth. A 363(1995)120.

9. S. Widdiyasekara, Initial velocity distributions of secondary ions in electronics sputtering of organic molecules, Ph.D. thesis, Uppsala University, 1987

10. G. såve, P. Håkansson, B.U.R. Sundqvist, U. Jöhnsson, G.Olofsson and M. malmquist, Spin-deposited submicrometer films of organic molecules for secondary ions mass spectrometry studies, Anal. Chem., 59, (1987) 2059 